\title{
Trauma-related dispatch criteria for Helicopter Emergency Medical Services in Europe
}

Laura D. Wigman, Esther M.M. van Lieshout, Gijs de Ronde, Peter Patka, Inger B. Schipper*

Department of Surgery-Traumatology, Erasmus MC, University Medical Center Rotterdam, the Netherlands

* Current address: Department of Surgery, Leiden University Medical Center, Leiden, the Netherlands

Correspondence address:

E.M.M. van Lieshout, $\mathrm{PhD}$

Erasmus MC, University Medical Center Rotterdam

Department of Surgery-Traumatology

P.O. Box 2040

3000 CA Rotterdam

The Netherlands

Phone: +31.10 .7031050$

Fax: +31.10 .7032396$

E-mail: e.vanlieshout@erasmusmc.nl

Keywords: Dispatch Criteria; Europe; HEMS; Helicopter Emergency Medical Services; Prehospital Care; Trauma; Trauma Helicopter 


\section{ABSTRACT}

Introduction: Helicopter Emergency Medical Services (HEMS) are used worldwide in order to provide potentially life-saving prehospital medical support to trauma patients at the accident scene. It is currently unclear how much overlap exists regarding the number and type of dispatch criteria used by individual HEMS organizations. The aim of the current study was to provide an overview of dispatch criteria for trauma cases used by HEMS organizations within Europe, and search for similarities and differences, between countries and HEMS stations.

Materials and methods: HEMS dispatch criteria related to trauma care were obtained from literature and divided into 4 groups of criteria and processed in a questionnaire. HEMS providing organisations were identified and contacted by telephone and via email.

Results: Fifty-five of the 65 organizations (85\%) that were contacted completed the questionnaire. The criteria "Fall from height", "Lengthy extrication and significant injury" and "Multiple casualty incidents" were used most frequently. Criteria from the subgroup "Patient characteristics - Co-morbidities and age" were used the least. In 44 of the organizations the Central Dispatch Centre (CDC) was primarily responsible for HEMS dispatch.

Conclusion: This overview demonstrates the lack of uniformity in the use of dispatch criteria for trauma assistance on a national and international level. Furthermore, the activation of HEMS is not only depending on dispatch criterion protocols, but is also influenced by organizational factors like the education of the dispatcher, the training of the EMS personnel, the familiarity with the dispatch criteria, and the responses of bystanders. Future research should aim to identify a general set of criteria with the highest discriminating potential. 


\section{INTRODUCTION}

"It is not the emergency patient who should be taken to the hospital to be seen by the doctor, but the hospital doctor should go out to see and treat the emergency patient at the scene of an accident” (Martin Kirschner 1879-1942) ${ }^{1}$. Martin Kirschner, a German surgeon who is wellknown to day for his remarkable innovations in many fields of medicine, including (orthopedic) trauma, already recognized the importance of pre hospital emergency medical services and the possible role for high velocity transport when he made this statement in 1938.

Two decades ago Helicopter Emergency Medical Services (HEMS) were limitedly available in Europe. Today these services have extended to 22 countries, and the number of countries with HEMS is still growing.

In the Netherlands HEMS have been introduced one decade ago. Although several studies have been performed demonstrating the beneficial effects of HEMS on survival of severely injured trauma patients ${ }^{2-8}$, HEMS remains subject for debate because of the high costs involved. In the Netherlands, the primary aim of HEMS is to bring specialized medical care to trauma patients at the accident scene. For logistical reasons, such as an extensive and well maintained high-way infrastructure, the majority of the patients (85-98\%) is transported to a hospital or trauma center by HEMS-physician assisted ground ambulances ${ }^{9}$.

Worldwide HEMS are dispatched for providing on-scene care to severely injured trauma patients based upon a set of dispatch criteria. These criteria should have high specificity and sensitivity in order to adequately identify the trauma patients that would benefit from HEMS assistance. Criteria that fail to identify patients that would benefit from HEMS assistance will lead to either overtriage and subsequently higher costs, or undertriage, which will deprive severely injured patients from getting urgently needed treatment that may potentially be life saving. Due to the evolutionary nature of symptoms following major trauma, and the fact that 
field triage is more often performed in the early stages of care, some mistriage is unavoidable ${ }^{10}$. The balance between overtriage and undertriage is very delicate. The American College of Surgeons (ACS) suggested that an overtriage rate up to $50 \%$ was to be expected in order to reduce the undertriage rate to $10 \%{ }^{11}$.

In 1990 the American College of Surgeons Committee on Trauma (ACSCOT) published field triage guidelines that had been developed to identify seriously injured patients, who would benefit the most by transport to a level 1 trauma centre ${ }^{12}$. In 1993 these criteria were revised due to the high costs and overtriage rate caused by the 1990 edition criteria.

The ACS triage criteria were divided into the following internationally accepted major subgroups ${ }^{13}$ : "Mechanism of Injury" (MOI), "Patient Characteristics - Anatomy", "Patient Characteristics- Physiologic Parameters", "Patient Characteristics - Co-morbidities and age", and "Transport Considerations". In general, the HEMS dispatch criteria are derived from these criteria, which were originally designed for field triage.

Little is known about the usage of the trauma-related HEMS dispatch criteria ${ }^{13}$ in different countries and HEMS stations throughout Europe. Therefore the aim of this study was to provide an overview of dispatch criteria related to trauma dispatch used by HEMS organizations in Europe, and search for similarities and differences, between countries and HEMS stations. 


\section{MATERIALS AND METHODS}

\section{Study period and setting}

The study took place at a level 1 Trauma center located in Rotterdam (The Netherlands) between April 1 and July 1, 2008.

\section{Collection of dispatch criteria and development of the questionnaire}

The dispatch criteria used in the survey were obtained from a recent review of Ringburg et $\mathrm{al}^{13}$, in which an extensive literature search in multiple databases was performed with the following search terms: (Air ambulances OR Aeromedical OR Air medical OR Emergency Medical Service OR Helicopter) and (Criteri OR Guideline OR Protocol OR Standard) and (Dispatch OR Deployment OR Triage Or Utilization).

In total 51 HEMS dispatch criteria related to trauma dispatches were obtained from 33 articles. These 51 criteria were subdivided into the groups of criteria as proposed by the ACS. The group "Mechanism of Injury" contained 20 criteria. The subgroups "Patient Characteristics Anatomy" and "Patients Characteristics - Physiologic Parameters" each contained 11 criteria. The subgroup "Patient Characteristics - Co-morbidities and Age" consisted of four criteria, and the remaining five criteria related to the subgroup "Transport Considerations". With these 51 dispatch criteria, a questionnaire was composed, in which the directors of all European HEMS stations were asked to state which of the criteria listed were applied by their station.

Two questions were added to the questionnaire in order to determine who was responsible for the actual dispatch of HEMS on a primary or secondary mission. No a priori definition of a central dispatch center (CDC) was provided. This could be either a national or regional EMS system including dispatch of HEMS, or the HEMS operators own dispatch system screening calls for help before dispatching the HEMS. A blank text field was provided for 
comments or remarks. The questionnaire was available on the hospital website for the duration of this study.

\section{Distribution of the questionnaire}

Names and addresses of HEMS organizations in Europe were collected from the Shepard handbook $^{14}$ and from the internet. All organizations that dispatched HEMS were considered eligible. Organizations only providing interhospital transportation or Search-And-Rescue activities were excluded.

A weblink to the questionnaire was sent to the supervisors of all organizations. If unclear from their website, organizations were contacted by telephone first, in order to obtain the name and e-mail address of the person best suited for further contact. Organizations that had not responded within two weeks received a reminder (by e-mail or telephone) every second week during the remainder of the study. The questionnaire could be completed on the internet, or could be returned to the principle investigator as an e-mail attachment. 


\section{RESULTS}

\section{Organizations}

After an intensive search in the Shepard handbook ${ }^{14}$ and on the internet, 77 HEMS organizations, representing over 300 helicopters in Europe, were identified. Twelve organizations in Italy could not be contacted due to outdated contact information $(\mathrm{N}=9)$ or linguistic problems $(\mathrm{N}=3)$. Fiftyfive of the 65 organizations $(85 \%)$ that were contacted completed the questionnaire. Of the 10 organizations that did not complete the questionnaire, two replied that they were unable to complete the questionnaire due to differences in the organization of the dispatch systems or the use of different dispatch criteria. The remaining 8 organizations were classified as nonresponders, after having received at least 3 reminders.

\section{All criteria combined}

Table 1 provides an overview of the number of organizations and countries that use the dispatch criteria. Six of the 55 organizations in 4 different countries (Poland, Sweden, Switzerland, UK) used all of the 51 dispatch criteria. Dispatch criteria of the group "Patient characteristics - Comorbidities and Age" (used by 11 to 13 countries, representing 19-21 organizations) is consistently used less than the other groups of dispatch criteria.

Table 2 lists how many dispatch criteria individual organizations use in each of the four criteria subgroups (i.e., MOI, type of injury, patient characteristics, and transport considerations). The smallest number of criteria was used by the London HEMS and the Scottish Ambulance Service, both located in the UK, which used only 6 and 7 of the listed criteria, respectively.

\section{Mechanism of Injury}

Seven of the twenty different criteria reflecting MOI were being used in all 19 responding countries (Table 2). The number of dispatch criteria related to MOI used by individual 
organizations ranged from 0 (Portugal) to 20 criteria (Austria, the Netherlands, Norway, Poland, Slovenia, Sweden, Switzerland, and UK).

The criteria "Fall from height", "Lengthy extrication and significant injury" and "Multiple casualty incidents" were used most frequently ( $\mathrm{N}=50$ organizations in $\mathrm{N}=19$ countries) (Table 1). The criterion "Significant displacement of front or rear axle" is the least frequently used criterion ( $\mathrm{N}=24$ organizations, $\mathrm{N}=11$ countries).

\section{Patient Characteristics - Anatomy}

The criterion "Multiple system injury" was being used as a dispatch criterion in 19 countries (Table 1). Twenty of the 55 organizations in ten countries used a total amount of 11 criteria from this group (Table 2). These represented Austria, Finland, Germany, Norway, Poland, Slovenia, Spain, Sweden, Switzerland, and the UK. The organizations Bomberos de Asturias (Spain) and the Scottish Ambulance Service (UK) did not use any of the dispatch criteria based on patient characteristics.

The most frequently used criterion was "Burns of significant Body Surface Area or relevant body regions"( $\mathrm{N}=49$ organizations from $\mathrm{N}=18$ countries), as opposed to "Finger/ thumb amputation", which was the least frequently used criterion ( $\mathrm{N}=30$ organizations in $\mathrm{N}=14$ countries).

\section{Patient Characteristics - Physiologic parameters}

The number of criteria used in this group ranges from 0 (Scottish ambulance service) to 11 (the Netherlands, Poland, Spain, Sweden, Switzerland, and the UK). The criterion "Anticipated need for ATLS procedures" was being used in 19 countries (Table 1). "Capillary refill" is the least frequently used criterion ( $\mathrm{N}=18$ organizations representing 11 countries). The most frequently 
used criterion is "Low Glasgow coma scale" $(\mathrm{N}=47$ organizations and it is being used in all of the countries with the exception of Luxembourg).

\section{Patient Characteristics - Co-morbidities and age}

In the subgroup "Co-morbidities and age" 13 organizations used all criteria as listed in the questionnaire. Twenty-seven organizations did not use any of these criteria (Table 1). The least used criterion was "Known respiratory disease" (N=19 organizations from 11 countries; Table 2).

\section{Transport considerations}

In the subgroup "Transport Considerations" the criterion "Inaccessible road/area" was being used in all the responding countries except for Luxembourg. ( $\mathrm{N}=50$ organizations from $\mathrm{N}=18$ countries). "Heavy traffic conditions" was the least frequently used criterion in this group. Twenty-six of the organizations used every criterion from this group for dispatching HEMS. Four organizations (OAMTC, Luxembourg Air Rescue, Alfa Helicopter, and London HEMS) did not use any of these criteria.

\section{Additional criteria}

Some organizations provided additional criteria. The OAMTC (Austria) is one of the organizations that uses additional criteria. These criteria are "Carbon monoxide intoxication with signs of compromised vital signs", "suffocation with respiratory compromise", "plain crash". But also criteria specified for mountain accidents such as "fall into glacier split" and "caught on safety lines after a fall'". The Acute Care Region East (Netherlands) also uses additional criteria;

"Train accidents", "plain crashes", "run over by a vehicle" and "paraplegia". 
In Slovakia "natuaral disaster", "transport of premature babies" and "perinatal with congenital defects of the heart" are also additional criteria for dispatching the HEMS.

\section{Dispatcher}

In 44 of the organizations the Central Dispatch Centre (CDC) was primarily responsible for HEMS dispatch (Table 2). In 4 of these CDCs a physician is responsible for the activation of HEMS. In the UK 4 organizations have a special HEMS desk or a HEMS paramedic that actively screens all emergency calls for the purpose of identifying calls that might meet HEMS dispatch criteria. In 3 organizations (Table 2; Acute Care Region East from the Netherlands, Christoph 6 Hradec Kralov from the Czech Republic, and HEMS Slovenia from Slovenia) HEMS are being dispatched by a ground ambulance paramedic or other first responding emergency-care provider. In two other organizations (Alfa Helicopter, Slovakia, and Bomberos de Asturias, Spain) a physician working for the HEMS organization is responsible for dispatching HEMS via radio communication. In two organizations (EMI from Portugal and Norwegian Air Ambulance Bergen) medical personnel on board of HEMS helicopter are used as dispatchers. 


\section{DISCUSSION}

The aim of this study was to provide an overview of trauma-related dispatch criteria used by HEMS providing organizations in Europe. This overview shows that dispatch criteria from the group "Mechanism Of Injury" are the most used criteria in Europe. It also reveals the differences in the use of dispatch criteria per country.

Individual criteria reflecting Mechanisms Of Injury were being used by 24 to 50 organizations, depending on the specific MOI. However, there are no organizations that solely use this group of criteria for dispatching their HEMS. Literature data revealed that criteria that are based upon MOI have a specificity varying between $72-97 \%^{15-18,13,19}$, implying that the overtriage rate (defined as 1-specificity) will be acceptable. Due to a poor sensitivity between 0-73\%, however, the undertriage rate (1-sensitivity) will be high. As a consequence, the majority of patients that would benefit from HEMS would be missed (and deprived of potentially life-saving treatment by a trauma team at the accident scene) when only using these criteria for HEMS dispatch. The general inability of dispatch criteria related to MOI for predicting severe injury has been described before ${ }^{10}$.

Fifty organizations in 18 countries used "penetrating injury" as a reason for dispatching HEMS, which might contradict with current belief that scoop and run is the best strategy for patients with penetrating injuries. In countries such as Austria and Finland where areas with mountains are present, or in countries with long distances to a hospital, sending a HEMS with advanced medical expertise to the accident scene, would be more favorable than the scoop and run strategy, as performed by an ground ambulance. 
The current study shows that dispatch criteria related to MOI and patient characteristics are frequently applied throughout Europe. Consequently, unacceptably high overtriage rates are at risk in almost every HEMS providing country in Europe. Although overtriage does not directly reduce patient safety, it results in overutilization of limited financial and human resources ${ }^{10}$.

The organizations Bomberos de Asturias (Spain) did not use any of the criteria within the group "Patients Characteristics - Anatomy". Instead, a physician working in a coordination centre has the authority to send HEMS based on his/her decision.

Regarding dispatch criteria based on physiological parameters, the outcome of this overview shows that between 24 and 47 organizations use these criteria with an average of 36 organizations per criteria. The Criterion "Low Glasgow coma scale" is an important criterion because it is a good indicator of the injury severity of a patient. It was to be expected that low GCS would the most used physiology criteria, as it is most likely the most appropriate indicator of patient status. Moront et $\mathrm{al}^{20}$ found a sensitivity of $98 \%$ and a specificity of $96 \%$ which suggest a high appropriateness of this criterion for HEMS dispatch. Ringburg et $\mathrm{al}^{13}$ concluded $^{13}$ that overall the criteria based on physiological parameters will result in little overtriage and moderate undertriage. Reliability is however influenced by the experience of the personnel at the scene, as also described previously ${ }^{10}$; if no paramedical personnel is available, physiological parameters can only be estimated, which will influence the sensitivity and specificity of these physiology based dispatch criteria. Nevertheless, they serve as one of the most adequate and useful, amongst the groups of dispatch criteria.

Criteria from the subgroup "Patient Characteristics - Co-morbidities and Age" were not being used in Italy, Luxembourg, Finland and Slovakia. Luxembourg provided additional information explaining that even though they used defined criteria for sending HEMS on a mission the 
dispatchers are allowed to make improvisations. Wuerz et al $^{21}$ found a sensitivity of $56 \%$ and a specificity of $45 \%$ for dispatch criteria from the subgroup "Patient Characteristics - Comorbidities and Age". Using these criteria will therefore lead to both under- and overtriage.

Dispatch criteria from the subgroup "Transport Considerations" are included in many dispatch protocols (i.e., in 13-18 countries and 28-50 organizations). These criteria mainly relate to logistics, and represent a separate entity. In general, bringing the specialized emergency physicians out to the patient is the main purpose of HEMS systems, but bringing the severely injured patient back to the hospital is also an important issue when ground transport is not possible or takes too much time. These considerations may differ between systems and countries, and may be influences by geography and population density.

Two organizations were not able to complete the questionnaire. These organizations are from Bulgaria and France. The Bulgarian organization "Air Ban Ltd" uses the interpretation of a specific situation by ambulance physicians as dispatchers for HEMS instead of predefined dispatch criteria. This system is also used in Slovenia. In France, the organization SAMU employs a physician in a dispatch center who is responsible for dispatching HEMS. These physicians use 6 reasons for dispatching HEMS and all other dispatches are based on individual decisions made by these physicians ${ }^{22}$.

Coats and Newton ${ }^{23}$ studied the dispatch system used by 4 organizations in the UK, where a special 'HEMS desk' within a control room staffed by paramedics was created. They found a significant reduction in non-required HEMS missions. A non-required mission was defined as a mission in which HEMS was dispatched but the patient was not treated, because the medical condition of the patient did not require HEMS treatment. The Yorkshire Air Ambulance 
organization, which also uses this dispatch system, found that their aborted missions dropped from $40 \%$ to $12 \%$, after implementation of this system.

The undertriage and overtriage rate of HEMS dispatch are mainly dependent upon the set of dispatch criteria used within the dispatch protocol. On the other hand, the dispatchers play a critical role in the execution of the protocol. A study by Gijsenbergh et $\mathrm{al}^{24}$ suggested that only repetitive training efforts could result in increased dispatching sensitivity without decreasing dispatch specificity.

Only few countries are involved in an international collaboration on the field of HEMS. These projects concern neighboring countries and their border area. The most recent project in Europe was the Cross-Border AIR Rescue project in Germany and Denmark ${ }^{25}$.

In the current survey, individual HEMS organizations were asked to indicate which HEMS dispatch criteria they applied for providing care to trauma patients at the accident scene. It is unclear to what extent the responders reflect their national system, as no further explanation or clarification was asked in the questionnaire. If countries had more than one HEMS organization, each individual organization was invited to participate (see Table 2).

The current survey was exclusively aimed at gaining insight into the variability in dispatch protocols used by HEMS systems throughout Europe. Whether or not the participating HEMS systems employed specialized emergency physicians capable of RSI endotracheal intubation, pleural drainage, and other life saving interventions was not part of this study. There is an ongoing debate if such interventions should be performed at the accident scene by a specialized physician or nurse ${ }^{26-28}$. 
Another limitation to the current study is that it was left to the discretion of the respondents to define whether or not a CDC was responsible for the dispatch decision. A CDC could be either national or regional EMS system including dispatch of HEMS, or the HEMS operators own dispatch system where calls for help are being screened before dispatching the HEMS. This may very between countries, and may be related to the payment system. It is unclear to what extent criteria meant to avoid overtriage may cause "missing" severely injured patients in the time window available. 


\section{CONCLUSION}

This overview demonstrates the lack of uniformity in the use of trauma-related dispatch criteria on a national and international level. The most frequently and uniformly used criteria for providing on-scene assistance to trauma patients in Europe are: "Fall from height", "Lengthy extrication and significant injury" and "Multiple casualty incidents". Activation of HEMS is not only depending on dispatch criterion protocols, but is also influenced by organizational factors like the education of the dispatcher, the training of the EMS personnel, the familiarity with the dispatch criteria, and the responses of bystanders. Currently, dispatch criteria based on the MOI and physiological parameters seem to be generally accepted as most suitable, with high specificity and intermediate sensitivity. Further research is needed in order to identify a general set of criteria with the highest discriminating potential, i.e., those that identify trauma patients that require HEMS assistance with both high sensitivity and specificity. Depending upon local circumstances, subsets of criteria might be subsequently added in order to further optimize the accuracy of the dispatch protocol per individual region.

\section{Conflict of Interest Statement}

All authors state that no conflict of interest, neither financial nor personal, exists. 


\section{ACKNOWLEDGEMENTS}

All HEMS organization representatives who completed our questionnaire and/or provided us with supplemental information are greatly acknowledged for providing us with information that has resulted in a more concise overview of HEMS usage within Europe. 


\section{REFERENCES}

1. Dick W. Resuscitation Great. Martin Kirschner: 1879-1942--a surgeon in prehospital care. Resuscitation 2006;68(3):319-321.

2. Biewener A., Aschenbrenner U., Rammelt S., et al. Impact of helicopter transport and hospital level on mortality of polytrauma patients. J Trauma 2004;56(1):94-98.

3. Brathwaite C.E., Rosko M., McDowell R., et al. A critical analysis of on-scene helicopter transport on survival in a statewide trauma system. J Trauma 1998;45(1):140-144; discussion 144-146.

4. Chappell V.L., Mileski W.J., Wolf S.E., Gore D.C. Impact of discontinuing a hospitalbased air ambulance service on trauma patient outcomes. J Trauma 2002;52(3):486-491.

5. $\quad$ Frankema S.P., Ringburg A.N., Steyerberg E.W., et al. Beneficial effect of helicopter emergency medical services on survival of severely injured patients. Br J Surg 2004;91(11):1520-1526.

6. $\quad$ Iirola T.T., Laaksonen M.I., Vahlberg T.J., Palve H.K. Effect of physician-staffed helicopter emergency medical service on blunt trauma patient survival and prehospital care. Eur J Emerg Med 2006;13(6):335-339.

7. $\quad$ Ringburg A.N., Thomas S.H., Steyerberg E.W., et al. Lives saved by helicopter emergency medical services: an overview of literature. Air Med J 2009;28(6):298-302.

8. Thomas S.H., Harrison T.H., Buras W.R., et al. Helicopter transport and blunt trauma mortality: a multicenter trial. J Trauma 2002;52(1):136-145.

9. Ringburg A.N., Frissen I.N., Spanjersberg W.R., et al. Physician-staffed HEMS dispatch in the Netherlands: Adequate deployment or minimal utilization? Air Med J 2005;24(6):248-251.

10. Rehn M., Eken T., Kruger A.J., et al. Precision of field triage in patients brought to a trauma centre after introducing trauma team activation guidelines. Scand J Trauma Resusc Emerg Med 2009;17(1):1.

11. Committee on Trauma. Resources for optimal care of the injured patient. In: American College of Surgeons 1998. Chicago; 1999.

12. Santaniello J.M., Esposito T.J., Luchette F.A., et al. Mechanism of injury does not predict acuity or level of service need: field triage criteria revisited. Surgery 2003;134(4):698703; discussion 703-694.

13. Ringburg A.N., de Ronde G., Thomas S.H., et al. Validity of helicopter emergency medical services dispatch criteria for traumatic injuries: a systematic review. Prehosp Emerg Care 2009;13(1):28-36.

14. Donaldson P. The Shepard Group's handbook of Public Service Aviation (Operator) Company listing: The Shepard Press Ltd 2006.

15. Bond R.J., Kortbeek J.B., Preshaw R.M. Field trauma triage: combining mechanism of injury with the prehospital index for an improved trauma triage tool. J Trauma 1997;43(2):283-287.

16. Cooper M.E., Yarbrough D.R., Zone-Smith L., et al. Application of field triage guidelines by pre-hospital personnel: is mechanism of injury a valid guideline for patient triage? Am Surg 1995;61(4):363-367.

17. Knudson P., Frecceri C.A., DeLateur S.A. Improving the field triage of major trauma victims. J Trauma 1988;28(5):602-606.

18. Norcross E.D., Ford D.W., Cooper M.E., et al. Application of American College of Surgeons' field triage guidelines by pre-hospital personnel. J Am Coll Surg 1995;181(6):539-544. 
19. Henry M.C., Hollander J.E., Alicandro J.M., et al. Incremental benefit of individual American College of Surgeons trauma triage criteria. Acad Emerg Med 1996;3(11):9921000 .

20. Moront M.L., Gotschall C.S., Eichelberger M.R. Helicopter transport of injured children: system effectiveness and triage criteria. J Pediatr Surg 1996;31(8):1183-1186; discussion 1187-1188.

21. Wuerz R.M. Accuracy of trauma triage in patients transported by helicopter. Air medical journal 1996;15(4):168-170.

22. Bertrand C., Dusseux E., Simon J.P., et al. Rapport sur l'étude de faisabilité d'une enquête nationale sur les transports héliportés. Médecine d'urgence 2002;24428-433.

23. Coats T.J., Newton A. Call selection for the Helicopter Emergency Medical Service: implications for ambulance control. J R Soc Med 1994;87(4):208-210.

24. Gijsenbergh F., Nieuwenhof A., Machiels K. Improving the first link in the chain of survival: the Antwerp experience. Eur J Emerg Med 2003;10(3):189-194.

25. www.crossborderairrescue.net. 11-05-5008.

26. Sollid S.J., Heltne J.K., Soreide E., Lossius H.M. Pre-hospital advanced airway management by anaesthesiologists: is there still room for improvement? Scand J Trauma Resusc Emerg Med 2008;16(1):2.

27. Sollid S.J., Lockey D., Lossius H.M. A consensus-based template for uniform reporting of data from pre-hospital advanced airway management. Scand J Trauma Resusc Emerg Med 2009;17(1):58.

28. Spanjersberg W.R., Ringburg A.N., Bergs E.A., et al. Prehospital chest tube thoracostomy: effective treatment or additional trauma? J Trauma 2005;59(1):96-101.

29. Indication for Air Medical Transport Activation. [cited; Available from: www.gov.ns.ca/health/ehs/Air_Ambulance.

30. Bamoski A., Kovach B., Podmore M., et al. Trauma triage: do AAMS transport guidelines do it effectively? Air Med J 1998;17(1):19-23.

31. Coats T.J., Wilson A.W., Cross F.W. On-scene medical decision making and overtriage. Br J Surg 1993;80(10):1291-1293.

32. O'Malley R.J., Watson-Hopkins M. Monitoring the appropriateness of air medical transports. Air Med J 1994;13(8):323-325.

33. Schoettker P. Ejection as a key word for the dispatch of a physician staffed helicopter: the swiss experience. Resuscitation 2001;49169-173.

34. Thomson D.P., Thomas S.H. Guidelines for air medical dispatch. Prehosp Emerg Care 2003;7(2):265-271.

35. Berns K.S. Use of the autolaunch method of dispatching a helicopter. Air medical journal 2003;22(3):35-41.

36. Wish J.R., Davis D.P. Auto launch/early activation: a survey of AAMS members and literature review. Air Med J 2005;24(2):83-88.

37. Garthe E., Mango N.K., Prenney B. Statewide air medical transports for Massachusetts. Air Med J 2002;21(6):22-28.

38. Garthe E.A., Mango N.K., Prenney B. A regional review of air medical transports for fatal motor vehicle crashes. Air Med J 2000;19(3):83-89.

39. Rhodes M., Perline R., Aronson J., Rappe A. Field triage for on-scene helicopter transport. J Trauma 1986;26(11):963-969.

40. Saffle J.R., Edelman L., Morris S.E. Regional air transport of burn patients: a case for telemedicine? J Trauma 2004;57(1):57-64; discussion 64.

41. Baack B.R., Smoot E.C., 3rd, Kucan J.O., et al. Helicopter transport of the patient with acute burns. J Burn Care Rehabil 1991;12(3):229-233. 
42. Kotch S.J., Burgess B.E. Helicopter transport of pediatric versus adult trauma patients. Prehosp Emerg Care 2002;6(3):306-308.

43. Norton R.M. Appropriate helicopter transport of urban trauma patients. The journal of trauma, injury, infection and critical care 1996;5886-891.

44. Diaz M.A., Hendey G.W., Bivins H.G. When is the helicopter faster? A comparison of helicopter and ground ambulance transport times. J Trauma 2005;58(1):148-153.

45. Slater H., O'Mara M.S., Goldfarb I.W. Helicopter transportation of burn patients. Burns 2002;28(1):70-72.

46. Cowley R.A., Hudson F., Scanlan E., et al. An economical and proved helicopter program for transporting the emergency critically ill and injured patient in Maryland. $\mathbf{J}$ Trauma 1973;13(12):1029-1038.

47. Holcomb J.B., Niles S.E., Miller C.C., et al. Prehospital physiologic data and lifesaving interventions in trauma patients. Mil Med 2005;170(1):7-13.

48. Holcomb J.B., Salinas J., McManus J.M., et al. Manual vital signs reliably predict need for life-saving interventions in trauma patients. J Trauma 2005;59(4):821-828; discussion 828-829.

49. Garner A., Lee A., Harrison K., Schultz C.H. Comparative analysis of multiple-casualty incident triage algorithms. Ann Emerg Med 2001;38(5):541-548.

50. Bledsoe B.E., Wesley A.K., Eckstein M., et al. Helicopter scene transport of trauma patients with nonlife-threatening injuries: a meta-analysis. J Trauma 2006;60(6):12571265; discussion 1265-1256.

51. Black J.J., Ward M.E., Lockey D.J. Appropriate use of helicopters to transport trauma patients from incident scene to hospital in the United Kingdom: an algorithm. Emerg Med J 2004;21(3):355-361.

52. Cocanour C.S., Fischer R.P., Ursic C.M. Are scene flights for penetrating trauma justified? J Trauma 1997;43(1):83-86; discussion 86-88.

53. Cocanour C.S., Ursic C., Fischer R.P. Does the potential for organ donation justify scene flights for gunshot wounds to the head? J Trauma 1995;39(5):968-970.

54. Davis D.P., Peay J., Serrano J.A., et al. The impact of aeromedical response to patients with moderate to severe traumatic brain injury. Ann Emerg Med 2005;46(2):115-122.

55. Position paper on the appropriate use of emergency air medical services. Association of Air Medical Services. J Air Med Transp 1990;9(9):29-30, 32-23.

56. Eckstein M., Jantos T., Kelly N., Cardillo A. Helicopter transport of pediatric trauma patients in an urban emergency medical services system: a critical analysis. J Trauma 2002;53(2):340-344.

57. Lerner E.B., Billittier A.J., Dorn J.M., Wu Y.W. Is total out-of-hospital time a significant predictor of trauma patient mortality? Acad Emerg Med 2003;10(9):949-954.

58. Petrie D.A., Tallon J.M., Crowell W., et al. Medically appropriate use of helicopter EMS: the mission acceptance/triage process. Air Med J 2007;26(1):50-54. 
Table 1 An overview of the dispatch criteria used within Europe

\section{Dispatch criterion:}

\section{Mechanism Of Injury:}

Fall from height $2,11,38,42,47,54,56,58$

Motor vehicle collision (MVC) with significant vehicle deformity ${ }^{3,55,56,58}$

Significant displacement of front or rear axle $2,38,56$

Significant passenger compartment intrusion on patient side, or on opposite side ${ }^{2,38,56}$

Patient ejected from vehicle $2,11,25,26,38,42,47,54,56,58$

Vehicle turnover $2,3,38,56$

Death same compartment $2,25,38,41,42,54,56$

Pedestrian struck $20 \mathrm{mph}^{2,3,11,25,26,38,42,54,56,58}$

Diving accident ${ }^{42,47}$

Electricity or lightning accident ${ }^{42,45,54}$

(Near) Drowning 3,42,54

Explosion ${ }^{42}$

Exposure to hazardous materials ${ }^{42}$

Fire in confined space, or inhalation injury ${ }^{1,42,45,54}$

Fatality on high speed roads ${ }^{3}$

Frontal collision on hardened roads outside urban area ${ }^{42}$

Lengthy extrication and significant injury 2, 3, 26, 33, 37, 38, 41, 42, 47, 55, 56

Logging/farm/industrial accidents 3,45

Multiple casualty incidents ${ }^{3}, 33,37,42,47,54,58$

Penetrating injury to head, neck, chest, abdomen or groin 2, 3, 11, 25, 38, 42, 54, 56, 58

Patient characteristics - Anatomy:

Two or more proximal long bone fractures, or open long bone fracture $11,42,54,56,58$

Burns of significant BSA or relevant body regions ${ }^{1-3,19,25,38,42,45,49,54,56,58}$

Flail chest ${ }^{54,56}$

Major proximal amputation or deglovement injury ${ }^{2,38,47,54,58}$

Skull fracture ${ }^{17,54,58}$

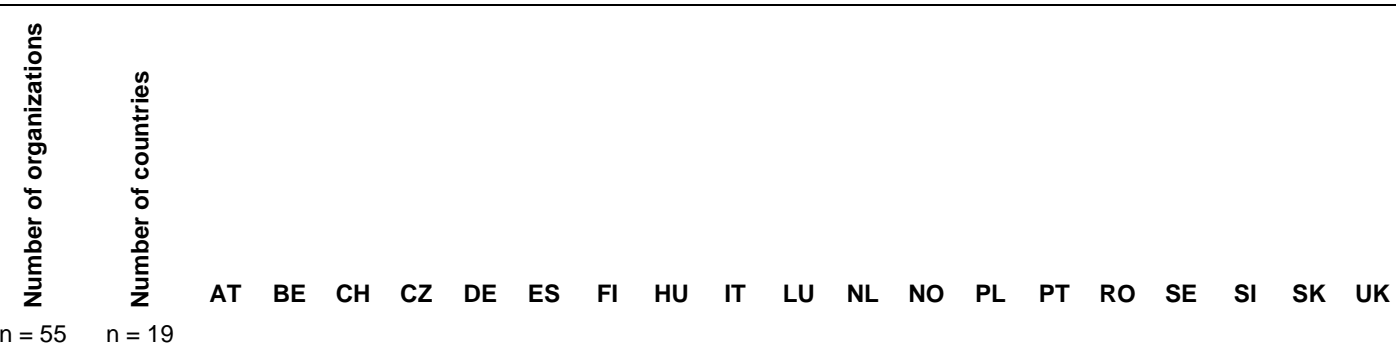

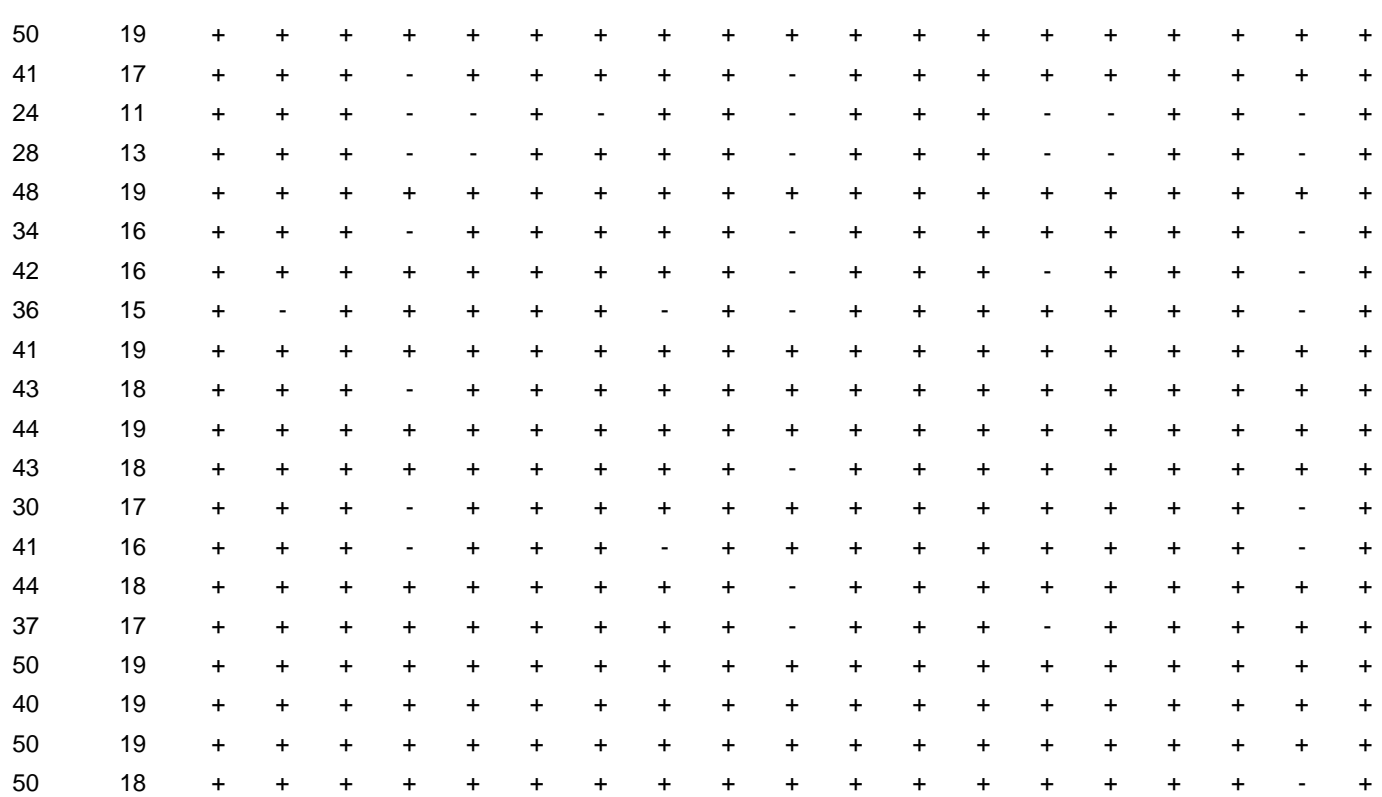

\begin{tabular}{llllllllllllllllllllllllll}
34 & 16 & + & + & + & + & + & + & + & + & + & - & + & + & + & - & + & + & + & - & + \\
49 & 18 & + & + & + & + & + & + & + & + & + & - & + & + & + & + & + & + & + & + & + \\
39 & 17 & + & + & + & + & + & + & + & + & + & - & + & + & + & + & + & + & + & - & + \\
46 & 18 & + & + & + & + & + & + & + & + & + & + & + & + & + & + & + & + & + & - & + \\
39 & 17 & + & + & + & + & + & + & + & + & + & - & + & + & + & + & + & + & + & - & + \\
\hline
\end{tabular}




\begin{tabular}{|c|c|c|c|c|c|c|c|c|c|c|c|c|c|c|c|c|c|c|c|c|c|}
\hline Finger/thumb amputation when emergent evaluation is indicated ${ }^{54}$ & 30 & 14 & + & + & + & - & + & + & + & - & - & + & + & + & + & - & - & + & + & + & + \\
\hline Fracture or dislocation with vascular compromise ${ }^{54,58}$ & 35 & 17 & + & + & + & + & + & + & + & + & + & - & + & + & + & + & + & + & + & - & + \\
\hline Extremity ischemia ${ }^{54}$ & 31 & 17 & + & - & + & + & + & + & + & + & + & - & + & + & + & + & + & + & + & + & + \\
\hline 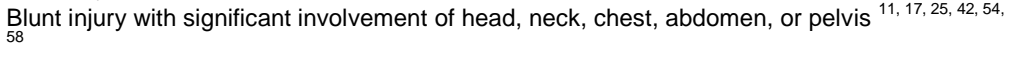 & 45 & 18 & + & + & + & + & + & + & + & + & + & - & + & + & + & + & + & + & + & + & + \\
\hline Multiple system injury $17,19,54$ & 47 & 19 & + & + & + & + & + & + & + & + & + & + & + & + & + & + & + & + & + & + & + \\
\hline Potential injury to spinal cord, or column $2,17,19,25,38,42,47,54,58$ & 44 & 18 & + & + & + & + & + & + & + & + & + & - & + & + & + & + & + & + & + & + & + \\
\hline \multicolumn{22}{|l|}{ Patient characteristics - Physiology: } \\
\hline Low or high respiratory rate or other signs of respiratory distress $2,11,17,25,38,41,42,45,56,58$ & 41 & 18 & + & + & + & + & + & + & + & + & + & - & + & + & + & + & + & + & + & + & + \\
\hline Low systolic blood pressure or tachycardia $^{2,11,17,25,29,30,35,38,41,42,54,56,58}$ & 36 & 17 & + & + & + & + & + & + & + & - & + & - & + & + & + & + & + & + & + & + & + \\
\hline Capillary refill > 2 seconds ${ }^{24}$ & 18 & 11 & - & - & + & + & - & + & + & - & - & - & + & + & + & - & + & + & + & - & + \\
\hline Unresponsive to verbal stimuli; Deteriorating mental status ${ }^{7,54}$ & 41 & 16 & + & + & + & - & + & + & + & + & + & - & + & + & + & + & + & + & + & - & + \\
\hline Low Circulation, respiration, abdomen, motor function and speech (CRAMS) score 2,38 & 26 & 14 & + & - & + & + & + & + & + & - & - & - & + & + & + & + & + & + & + & - & + \\
\hline Low Glasgow coma scale $2,11,25,29,30,35,38,41,42,54,56,58$ & 47 & 18 & + & + & + & + & + & + & + & + & + & - & + & + & + & + & + & + & + & + & + \\
\hline Paramedic judgment/intuition $6,14,13,18,54,55,58$ & 45 & 18 & + & + & + & + & + & + & + & + & + & + & + & + & + & - & + & + & + & + & + \\
\hline Post-traumatic cardiac arrest ${ }^{17}$ & 43 & 17 & + & + & + & + & + & + & + & - & + & + & + & + & + & + & + & + & + & - & + \\
\hline Low (Revised) Trauma score $2,38,42,54,55$ & 32 & 14 & + & - & + & + & + & + & + & + & + & - & + & - & + & + & - & + & + & - & + \\
\hline Algorithms: Modified simple Triage and rapid treatment; Triage Sieve; Care Flight Triage ${ }^{24}$ & 24 & 13 & + & - & + & + & + & + & - & + & + & - & + & + & + & + & - & + & - & - & + \\
\hline Anticipated need for ATLS procedures ${ }^{18,48,54}$ & 43 & 19 & + & + & + & + & + & + & + & + & + & + & + & + & + & + & + & + & + & + & + \\
\hline \multicolumn{22}{|l|}{ Patient characteristics - Co-morbidities and age: } \\
\hline Age $<5 y$ or $>55 y^{2}, 25,38,47,54,56$ & 21 & 12 & - & - & + & + & + & + & - & - & - & - & + & + & + & + & + & + & + & - & + \\
\hline Known cardiac disease ${ }^{19,25,45,55,56}$ & 20 & 11 & + & - & + & - & + & + & - & - & - & - & + & - & + & + & + & + & + & - & + \\
\hline Known respiratory disease $\mathrm{I}^{19,25,45,55,56}$ & 19 & 11 & - & - & + & + & + & + & - & - & - & - & + & - & + & + & + & + & + & - & + \\
\hline Known pregnancy ${ }^{25,54,58}$ & 21 & 13 & - & + & + & + & + & + & - & + & - & - & - & + & + & + & + & + & + & - & + \\
\hline \multicolumn{22}{|l|}{ Transport considerations: } \\
\hline Expectation of prolonged transport time ${ }^{14,13,18,19,22,25,34,39,41,47,48,54-56}$ & 44 & 17 & + & + & + & + & + & + & + & - & + & - & + & + & + & + & + & + & + & + & + \\
\hline Inaccessible road/area ${ }^{3,6,25,33,37,39,41,48,54-56}$ & 50 & 18 & + & + & + & + & + & + & + & + & + & - & + & + & + & + & + & + & + & + & + \\
\hline Heavy traffic conditions ${ }^{14,13,25,33,37,48}$ & 28 & 13 & - & + & + & + & + & + & + & - & - & - & + & + & + & - & + & + & + & - & + \\
\hline Distance to trauma center ${ }^{54}$ & 46 & 18 & + & + & + & + & + & + & + & + & + & - & + & + & + & + & + & + & + & + & + \\
\hline Prolonged prehospital time ${ }^{14,13,18,19,22,25,34,39,41,47,48,54-56}$ & 37 & 15 & + & + & + & + & + & + & + & - & + & - & + & + & + & - & + & + & + & - & + \\
\hline
\end{tabular}

+ , country that uses the dispatch criterion specified; -, country that does not use this dispatch criterion; At, Austria; Be, Belgium; CH, Switzerland; CZ, Czech Republic; DE, Germany; ES, Spain; FI, Finland; HU, Hungary; IT, Italy; LU, Luxembourg; NL, Netherlands; NO, Norway, PL, Poland; PT, Portugal; RO, Romania; SE, Sweden; SI, Slovenia; SK, Slovakia ; UK, United Kingdom 
Table 2 The number of dispatch criteria used per organization

\begin{tabular}{|c|c|c|c|c|c|c|c|c|}
\hline 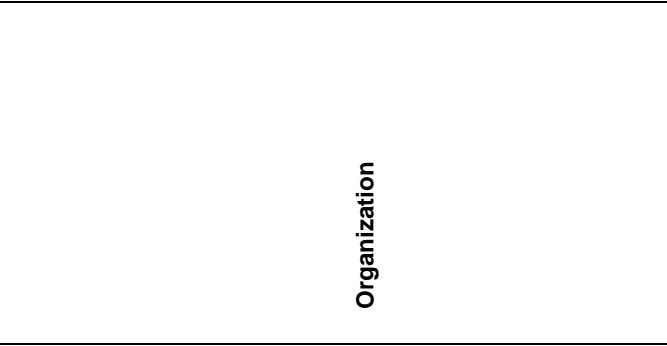 & 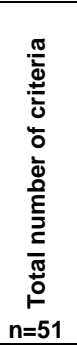 & $\sum_{n=20}^{\bar{O}}$ & 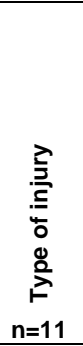 & 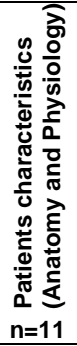 & 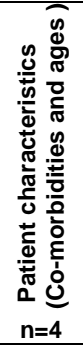 & 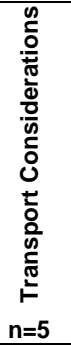 & 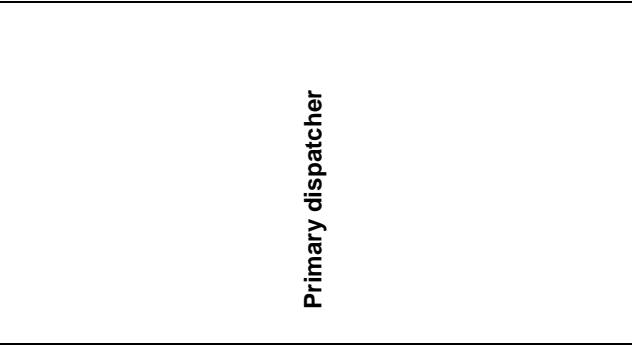 & 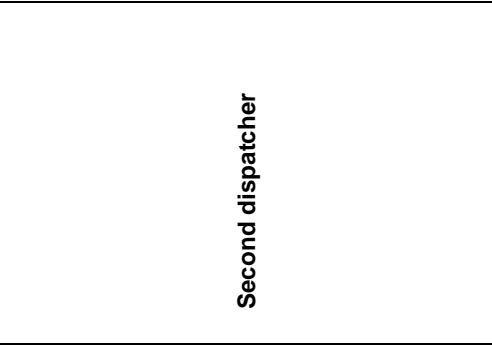 \\
\hline Austria & 46 & 20 & 11 & 10 & 1 & 4 & & \\
\hline ARA Flugrettungs- GmbH & 45 & 20 & 11 & 9 & 1 & 4 & CDC & GA paramedic/ 1st responding care provider \\
\hline OAMTC & 19 & 11 & 2 & 6 & 0 & 0 & $\mathrm{CDC}$ & GA paramedic/1st responding care provider \\
\hline Belgium & 42 & 19 & 11 & 7 & 1 & 5 & & \\
\hline MUG Brugge & 29 & 13 & 7 & 7 & 0 & 2 & CDC & GA paramedic or family doctor \\
\hline Centre médicalisé de secours de Bra & 38 & 18 & 8 & 6 & 1 & 5 & $\mathrm{CDC}$ & \\
\hline Czech Republic & 41 & 13 & 10 & 10 & 3 & 5 & & \\
\hline Territorial Rescue Centre of Moravian-Silesian Region & 37 & 9 & 10 & 10 & 3 & 5 & CDC & Physician working for HEMS organization \\
\hline Christoph 6 Hradec Kralove & 19 & 8 & 3 & 4 & 0 & 4 & GA paramedic/ 1st responding care provider & \\
\hline Finland & 45 & 19 & 11 & 10 & 0 & 5 & & \\
\hline Medi-Heli, Helsinki & 25 & 11 & 6 & 5 & 0 & 3 & CDC & GA paramedic/ 1st responding care provider \\
\hline Pelastushelikopteri SEPE & 44 & 19 & 11 & 9 & 0 & 5 & CDC & Physician working for HEMS organization \\
\hline IImari Eastern Finland HEMS & 31 & 16 & 6 & 5 & 0 & 4 & $\mathrm{CDC}$ & GA paramedic/ 1st responding care provider \\
\hline Rescue Helicopter ASLAK & 33 & 17 & 8 & 5 & 0 & 3 & $\mathrm{CDC}$ & GA paramedic/ 1st responding care provider \\
\hline Germany & 48 & 18 & 11 & 10 & 4 & 5 & & \\
\hline ADAC: Federal Armed Forces Medical Centre Ulm & 48 & 18 & 11 & 10 & 4 & 5 & CDC & GA paramedic/ 1st responding care provider \\
\hline Luxembourg Air Rescue & 41 & 18 & 10 & 8 & 0 & 5 & CDC & \\
\hline Hungary & 38 & 18 & 10 & 7 & 1 & 2 & & \\
\hline National Air Ambulance Public Company & 38 & 18 & 10 & 7 & 1 & 2 & $\mathrm{CDC}$ & GA paramedic/ 1st responding care provider \\
\hline Italy & 43 & 20 & 10 & 9 & 0 & 4 & & \\
\hline 118 HEMS - Udine & 37 & 18 & 10 & 7 & 0 & 2 & CDC & \\
\hline S.S.U.Em 118 Milano & 37 & 19 & 8 & 7 & 0 & 3 & CDC & \\
\hline 118 Piemonte EMS & 30 & 13 & 8 & 6 & 0 & 3 & CDC & \\
\hline 118 Firenze Soccorso & 33 & 19 & 4 & 7 & 0 & 3 & CDC & \\
\hline Luxembourg & 17 & 11 & 3 & 3 & 0 & 0 & & \\
\hline Luxembourg Air Rescue & 17 & 11 & 3 & 3 & 0 & 0 & dispatcher in combination with physician from EMS crew & \\
\hline The Netherlands & 50 & 20 & 11 & 11 & 3 & 5 & & \\
\hline Trauma Centre North West (Life Liner 1) & 33 & 15 & 8 & 8 & 0 & 2 & CDC & GA paramedic/ 1st responding care provider \\
\hline Trauma Centre South West (Life Liner 2) & 45 & 20 & 8 & 11 & 1 & 5 & CDC & GA paramedic/ 1st responding care provider \\
\hline Acute Care Region East (Life Liner 3) & 23 & 11 & 5 & 6 & 0 & 1 & GA paramedic/ 1st responding care provider & MP on board of HEMS helicopter \\
\hline Trauma Centre North (Life Liner 4) & 46 & 20 & 10 & 9 & 3 & 4 & $\mathrm{CDC}$ & GA paramedic/ 1st responding care provider \\
\hline Norway & 49 & 20 & 11 & 10 & 2 & 5 & & \\
\hline Norwegian Air Ambulance Ldt & 39 & 17 & 9 & 8 & 2 & 3 & CDC & MP on board of HEMS helicopter \\
\hline Norwegian Air Ambulance Bergen & 43 & 20 & 11 & 5 & 2 & 5 & MP on board of HEMS helicopter & Physician (general practitioner) on scene \\
\hline Poland & 51 & 20 & 11 & 11 & 4 & 5 & & \\
\hline Polish Medical Air Rescue & 51 & 20 & 11 & 11 & 4 & 5 & CDC & Physician in receiving hospital \\
\hline
\end{tabular}




\begin{tabular}{|c|c|c|c|c|c|c|c|c|}
\hline Portugal & 41 & 16 & 9 & 9 & 4 & 3 & & \\
\hline EMI & 19 & 1 & 4 & 9 & 3 & 2 & MP on board of HEMS helicopter & \\
\hline INEM & 37 & 16 & 9 & 6 & 3 & 3 & Physician in the CDC & \\
\hline Romania & 46 & 18 & 10 & 9 & 4 & 5 & & \\
\hline SMURD & 46 & 18 & 10 & 9 & 4 & 5 & $\mathrm{CDC}$ & MP on board of HEMS helicopter \\
\hline Slovenia & 50 & 20 & 11 & 10 & 4 & 5 & & \\
\hline HEMS Slovenia & 50 & 20 & 11 & 10 & 4 & 5 & GA paramedic/ 1st responding care provider & Physician in receiving hospital \\
\hline Slovakia & 26 & 12 & 6 & 5 & 0 & 3 & & \\
\hline AIR TRANSPORT EUROPE sr.o. & 23 & 12 & 5 & 3 & 0 & 3 & CDC & MP on board of HEMS helicopter \\
\hline Alfa helicopter & 6 & 2 & 2 & 2 & 0 & 0 & Physician working for HEMS organization & Insurance company physician \\
\hline Spain & 50 & 20 & 11 & 11 & 4 & 5 & & \\
\hline COYOTAIR S.A. - CASTILLA Y LEON & 48 & 18 & 11 & 10 & 4 & 5 & CDC & MP on board of HEMS helicopter \\
\hline RACC Serveis Mèdics & 34 & 15 & 8 & 7 & 0 & 4 & Physician in the CDC & Physician in a ground ambulance \\
\hline Servicio de Urgencias Canario & 49 & 18 & 11 & 11 & 4 & 5 & Physician in the CDC & Physician in a ground ambulance \\
\hline SUMMA 112 & 23 & 2 & 11 & 5 & 0 & 5 & $\mathrm{CDC}$ & 2nd dispatcher controlling ALS fleet \\
\hline SEM catalonia & 34 & 16 & 8 & 5 & 0 & 5 & $\mathrm{CDC}$ & Physician working for HEMS organization \\
\hline Bomberos de Asturias & 8 & 1 & 0 & 2 & 0 & 5 & Physician working for HEMS organization & GA paramedic/1st responding care provider \\
\hline Emprese Publica De Emergencias Sanitaria (EPES) & 40 & 19 & 6 & 9 & 2 & 4 & Physician in the CDC & \\
\hline 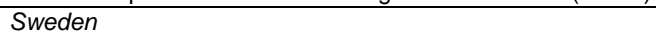 & 51 & 20 & 11 & 11 & 4 & 5 & & \\
\hline Uppsala Air ambulance helicopter & 22 & 13 & 3 & 4 & 0 & 2 & CDC & \\
\hline Ambulanshelikoptern VGR & 36 & 13 & 11 & 6 & 1 & 5 & $\mathrm{CDC}$ & GA paramedic/ 1st responding care provider \\
\hline Scandinavian Medicopter AB lufttranssport & 51 & 20 & 11 & 11 & 4 & 5 & $\mathrm{CDC}$ & \\
\hline Switzerland & 51 & 20 & 11 & 11 & 4 & 5 & & \\
\hline Rega & 51 & 20 & 11 & 11 & 4 & 5 & $\mathrm{CDC}$ & Physician working for HEMS organization \\
\hline Air Zermatt & 43 & 17 & 11 & 8 & 2 & 5 & $\mathrm{CDC}$ & Ground operation dispatcher on HEMS base \\
\hline United Kingdom & 51 & 20 & 11 & 11 & 4 & 5 & & \\
\hline Scottisch Ambulance Service & 6 & 2 & 0 & 0 & 0 & 4 & $\mathrm{CDC}$ & GA paramedic/ 1st responding care provider \\
\hline Essex Ambulance Service & 49 & 18 & 11 & 11 & 4 & 5 & $\mathrm{CDC}$ & \\
\hline London HEMS/London Royal & 7 & 5 & 1 & 1 & 0 & 0 & HEMS paramedic at CDC & GA paramedic/ 1st responding care provider \\
\hline County Air Ambulance & 51 & 20 & 11 & 11 & 4 & 5 & $\mathrm{CDC}$ & GA paramedic/ 1st responding care provider \\
\hline GNAAS(Great North Air Ambulance) & 17 & 8 & 4 & 1 & 0 & 4 & $\mathrm{CDC}$ & GA paramedic/ 1st responding care provider \\
\hline Yorkshire Air Ambulance & 50 & 19 & 11 & 11 & 4 & 5 & Dedicated Air Desk Ambulance Service & \\
\hline Kent Surrey Sussex Air Ambulance & 28 & 13 & 8 & 5 & 0 & 2 & special dedicated HEMS desk inside CDC & \\
\hline Wiltshire Air Ambulance & 51 & 20 & 11 & 11 & 4 & 5 & $\mathrm{CDC}$ & GA paramedic/1st responding care provider \\
\hline Lincolnshire \& Nottinghamshire Air Ambulance & 50 & 20 & 11 & 11 & 3 & 5 & $\mathrm{CDC}$ & GA paramedic/ 1st responding care provider \\
\hline Warwickshire \& Northamptonshire Air Ambulance & 30 & 11 & 8 & 5 & 1 & 5 & $\mathrm{CDC}$ & GA paramedic/ 1st responding care provider \\
\hline Devon Air Ambulance Trust & 46 & 20 & 11 & 11 & 0 & 4 & $\mathrm{CDC}$ & GA paramedic/1st responding care provider \\
\hline Cornwall & 51 & 20 & 11 & 11 & 4 & 5 & HEMS dispatcher CDC & GA paramedic/ 1st responding care provider \\
\hline
\end{tabular}

CDC, Central Dispatch Center; GA, Ground Ambulance; MP, Medical Personnel; EMS, Emergency Medical Services; HEMS, Helicopter Emergency Medical Services. 